\title{
Optimal placement of a limited number of observations for period searches
}

\author{
E. S. Saunders, T. Naylor, and A. Allan
}

\author{
School of Physics, University of Exeter, Stocker Road, EX4 4QL, UK \\ e-mail: [saunders,timn, aa]@astro.ex.ac.uk
}

Received 23 December 2005 / Accepted 13 April 2006

\begin{abstract}
Robotic telescopes present the opportunity for the sparse temporal placement of observations when period searching. We address the best way to place a limited number of observations to cover the dynamic range of frequencies required by an observer. We show that an observation distribution geometrically spaced in time can minimise aliasing effects arising from sparse sampling, substantially improving signal detection quality. The base of the geometric series is however a critical factor in the overall success of this strategy. Further, we show that for such an optimal distribution observations may be reordered, as long as the distribution of spacings is preserved, with almost no loss of quality. This implies that optimal observing strategies can retain significant flexibility in the face of scheduling constraints, by providing scope for on-the-fly adaptation. Finally, we present optimal geometric samplings for a wide range of common observing scenarios, with an emphasis on practical application by the observer at the telescope. Such a sampling represents the best practical empirical solution to the undersampling problem that we are aware of. The technique has applications to robotic telescope and satellite observing strategies, where target acquisition overheads mean that a greater total target exposure time (and hence signal-to-noise) can often in practice be achieved by limiting the number of observations.
\end{abstract}

Key words. methods: observational - methods: data analysis - techniques: photometric - techniques: radial velocities

\section{Introduction}

Recent years have seen the rise of research-grade robotic telescopes such as the Liverpool Telescope (Bode et al. 1999), as well as a sharp increase in the total number of autonomous telescopes active across the world (Hessman et al. 2004). The emergence of these telescopes and the flexibility of their operation justifies renewed interest in the optimum scheduling of observations. Our aim is to discuss in detail one such strategy and to demonstrate its optimal properties for our project. Individual robotic facilities offer new scheduling possibilities to the observer by relaxing the requirement that a human be physically present at the telescope at all times. Robotic telescopes offer the potential for ideal sampling of periodic signals, since observations may be requested more sparsely and over a longer timescale than a traditional continuous observing run.

Furthermore, when unmanned telescopes are linked by a communication infrastructure, new ways of performing science become feasible (Allan et al. 2004). For example, the diurnal sampling problems that plague single-site observations of periodic variables can be avoided if an observer can acquire data from a second, geographically distant site. Continuous monitoring projects such as the Whole Earth Telescope (Nather et al. 1990), and the BiSON network for solar observations (Chaplin et al. 1996) indicate the success of this approach.

Thus we arrive at a central question in the study of periodic stars: given access to round-the-clock observing, how can we best sample a potentially periodic star, whose luminosity oscillates with some unknown frequency, such that we can minimise the effects of aliasing, while providing a suitably long baseline with which to acquire the periods of interest, all while maintaining the quality of our data? In particular, how should we choose when to observe in those situations where the total number of observations that may be made is a limited resource? This situation is commonly the case for robotic telescopes, where target acquisition overheads, which include the slew time and the CCD readout time, as well as scheduler requirements, can preclude large numbers of observations. Although this question reflects our interests in using robotic networks to study variable stars, it is equally applicable to satellite-based observations.

The astrophysics of the problem limits the frequencies of interest to an astronomer, allowing limits to be placed on the range of frequencies which must be correctly reconstructed. Then for an evenly sampled dataset, the input signal may be correctly reconstructed if the sampling frequency is at least twice the highest frequency $v_{\max }$ to be detected. Assuming the astronomer wishes to see two cycles of any modulation then the lowest frequency detectable is given by $2 / T$, where $T$ is the duration of the total sampling interval (i.e. the run-length). The value of the required sampling frequency $v_{\mathrm{N}}$ for equally spaced data can in some way be viewed as the Nyquist frequency of the dataset, given by

$v_{\max }<N / 2 T=v_{\mathrm{N}}$

where $N$ is the number of observations. The problem becomes more interesting, however, for the case in which there are not sufficient datapoints to fulfill this condition. Then the question is whether, under these conditions, we can sample in such a way that we can recover a signal of acceptable quality. Alternatively, we can reverse the problem: how many datapoints do we need in order to recover an acceptable signal? In what follows, we show that there is scope for significant optimisation of observation placement in this undersampled regime. 


\section{Previous work}

In the field of time domain astronomy, there is a relative dearth of literature regarding the best way to sample data in time. In contrast, much attention has been focussed on the problem of signal extraction from fixed, finite datasets (e.g. Horne \& Baliunas 1986; Schwarzenberg-Czerny 2003). This is perhaps unsurprising. The vast majority of such datasets are acquired in the classical situation of a single extended observing run, where an astronomer physically travels to a remote observing site, accumulating data continuously each night for the duration of the run. In this case the observer has relatively little choice about how to space the observations, and normally opts for the safe strategy of using all of the available time for continuous observing, acquiring a large total number of observations.

Deeming (1975) showed that the observed Fourier transform of a discrete dataset can be expressed as the convolution of the true Fourier transform $F_{\mathrm{N}}(v)$ with a spectral window $\delta_{\mathrm{N}}(v)$ that fully describes the interference effects between the sampled frequencies, such that

$F_{\mathrm{N}}(v)=F(v) * \delta_{\mathrm{N}}(v)$,

where $F(v)$ is the complex Fourier transform of a function $f(t)$ and is defined as

$F(v)=\int_{-\infty}^{+\infty} f(t) \mathrm{e}^{\mathrm{i} 2 \pi v t} \mathrm{~d} t$,

and the spectral window is given by

$\delta(v)=\sum_{k=1}^{N} \mathrm{e}^{\mathrm{i} 2 \pi v t_{k}}$,

where $N$ is the total number of observations and $t_{k}$ is the set of observation times. The window function is even, so only positive frequencies are evaluated. The physical interpretation of the window function can be seen by considering the Fourier transform of an infinite sine function (a delta function) convolved with the window function, leading to the observed Fourier transform. Adjusting the set of observation times $t_{k}$ alters the window function, and hence the Fourier transform. Deeming (1975) showed that aliasing effects in the observed Fourier transform arising from the window function could be mitigated by introducing irregularities into the sampling pattern. By careful choice of sampling it is possible to reconstruct higher frequencies than $v_{\mathrm{N}}$, the highest frequency that could be accurately reconstructed if the same number of observations were equally spaced.

Deeming (1975) illustrated the behaviour of the window function through the use of a simple single-variable model for the $N=25$ case, empirically deriving a form for the spacing based on visual inspection of the resulting spectral window function. Mould et al. (2000) adopted a pseudo-geometric spacing as a way to maximise the uniformity of their phase spacing, but no details of the implemented optimisation strategy were provided.

The Rayleigh resolution criterion may be written as $\Delta v \Delta T=1$. It means that observations of length $\Delta T$ yield the frequency of an oscillation with an error of $\Delta v=1 / \Delta T$, at worst. After an additional time interval $\alpha \Delta T$ this yields an error in the cycle count $\Delta v \alpha \Delta T=\alpha$. Thus to keep the cycle count error less than $\alpha$, subsequent observations ought to be scheduled no later than $(1+\alpha) \Delta T$ from the start. Recurrent and rigid application of this known principle yields a geometric series scheduling (we are grateful to the referee for suggesting this line of argument).
We shall show that the choice of geometric base is crucial for such a strategy to succeed. The general question of what form an optimal uneven spacing should take, when the number of observations and the length of the observing run are free parameters remains, to our knowledge, unanswered.

Here we present a detailed analysis of the application of a geometric spacing technique to the undersampling problem. Although our sampling is not an analytic solution to the general problem of arbitrary placement of the dataset $t_{k}$, it outperforms both the model of Deeming (1975) and pure random distributions, and thus represents the best current heuristic solution to the sampling problem. In Sect. 3, we present the sampling strategy and quality metrics by which it is judged. In Sect. 4 we compare the geometric sampling to the sampling of Deeming (1975) and to random sampling and show that noise has no impact on the sampling strategy. We also show that the window function retains similar characteristics under random reordering of the spacings, an important result for practical scheduling scenarios. In Sect. 5 we present a graph indicating optimal geometric samplings for observing runs spanning the range from 10 to $500 \mathrm{ob}-$ servations. We present our final conclusions in Sect. 6.

\section{Method}

We generate our observation times with a geometric distribution using the scheme

$t_{k}=\frac{x^{k}-1}{x^{(N-1)}-1} T$,

where $t_{k}$ is the (time) value of the point in the series, $x$ is the base of the series, $k$ is the number of the current data point, $N$ is the total number of observations and $T$ is the duration of the total observing interval. This produces a geometrically spaced distribution such that $0 \leq t_{k} \leq T$.

The parameter $x$, which we call the geometric base, may be arbitrarily chosen. Since the window function is in general complex, for comparison with Deeming (1975) we follow the convention of examining the normalised amplitude $A(v)$, defined as the square of the amplitude of the window function, normalised by the square of the number of datapoints, such that

$A(v)=\frac{|\delta(v)|^{2}}{N^{2}}$

We define the optimal base, $x_{\mathrm{opt}}$, as the value for which the overall degree of structure present in $A(v)$ is minimised. We use the root mean square (rms) of $A(v)$ as a straightforward metric for identifying structure. Minimising the rms thus represents the desire of the astronomer to achieve relatively even sensitivity to periods.

In order to retain sensitivity to the higher frequency structure we evaluate $A(v)$ for frequencies in the range $0.1<v_{\mathrm{N}} \leq 5$ (in units of the Nyquist frequency for an equivalent even sampling). In practice, the range of the window function that needs to be considered will vary depending on the degree of undersampling of a given dataset (which depends on the number of observations and the length of the dataset), and the maximum frequency to be detected. Since what matters is the frequency with respect to the total time interval, we define the dimensionless relative frequency as

$v_{\text {rel }}=v T$.

Then the relative limiting frequency in units of the relative Nyquist frequency is simply $v_{\max } / v_{\mathrm{N}}$. 


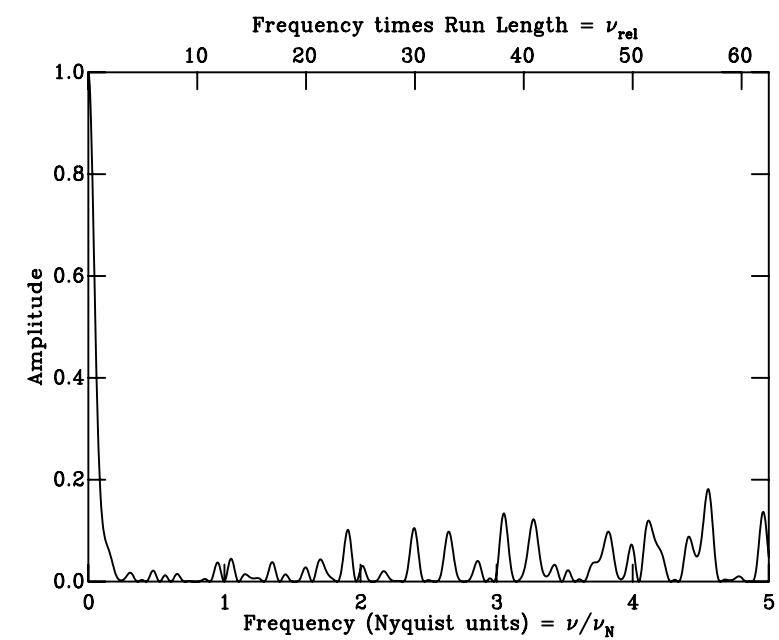

Fig. 1. The window function (where the amplitude is given by $\left.|\delta(v)|^{2} / N^{2}\right)$ produced by Deeming's distribution, with a close to optimal value of $\alpha=1.0$. The rms is 0.0361 .

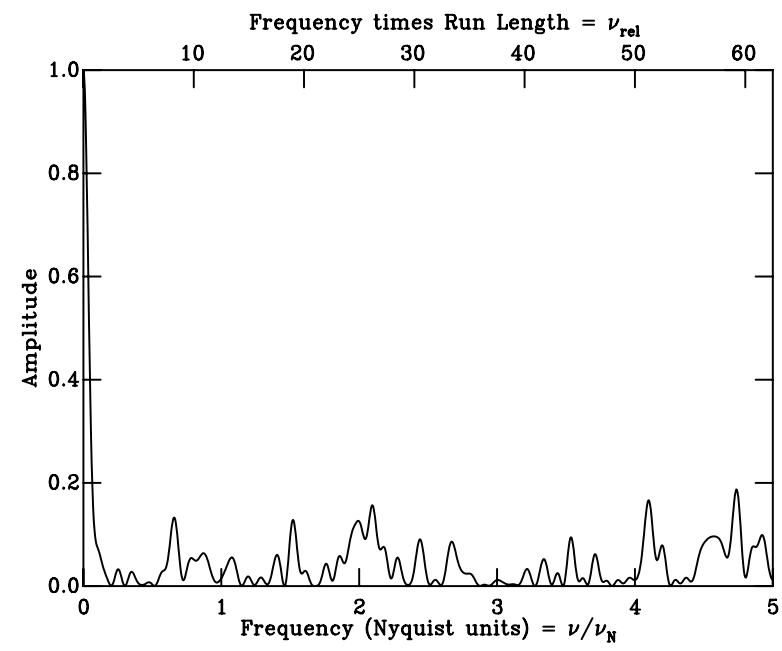

Fig. 2. The window function for a typical random distribution of 25 data points. The rms is 0.0386 .

\section{Results}

\subsection{The best sampling}

Deeming (1975) generated 25 datapoints according to the formula

$t \sim \begin{cases}k^{-1 / \alpha} & (k=1 \ldots 12) \\ (25-k)^{-1 / \alpha} & (k=13 \ldots 24) .\end{cases}$

Figure 1 is the window function for Deeming's distribution with $\alpha=1.0-$ a near-optimal value by visual inspection and rms comparison. Figure 2 presents the window function for a randomly spaced set of 25 datapoints. The typical size of the interference structures at high frequency are comparable, as indicated by the similar rms values of the two graphs. Figure 3 is the window function for an optimal geometric spacing, where the value of $x$ was chosen according to the minimum of Fig. 4 . The geometric spacing exhibits much better high frequency suppression, with an rms roughly half that of either of the other spacings, at the price of a slightly broader true peak (centred by construction at $v=0$ ). This broadening is a consequence of the undersampling. Although we can push the effective limit for frequency reconstruction to higher frequencies, we sacrifice some of our

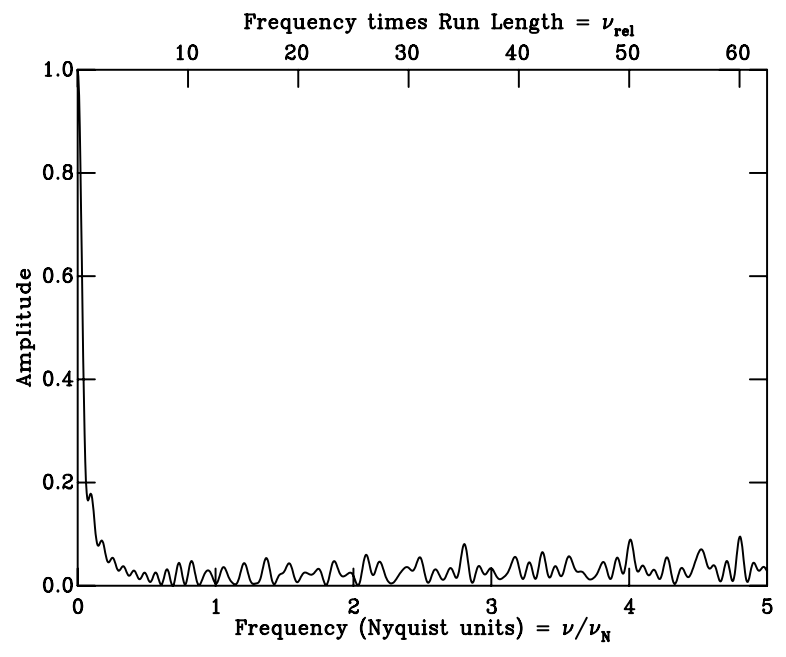

Fig. 3. The window function for the optimum geometric sampling of 25 data points, for $x=1.124$. The rms is 0.0201 .

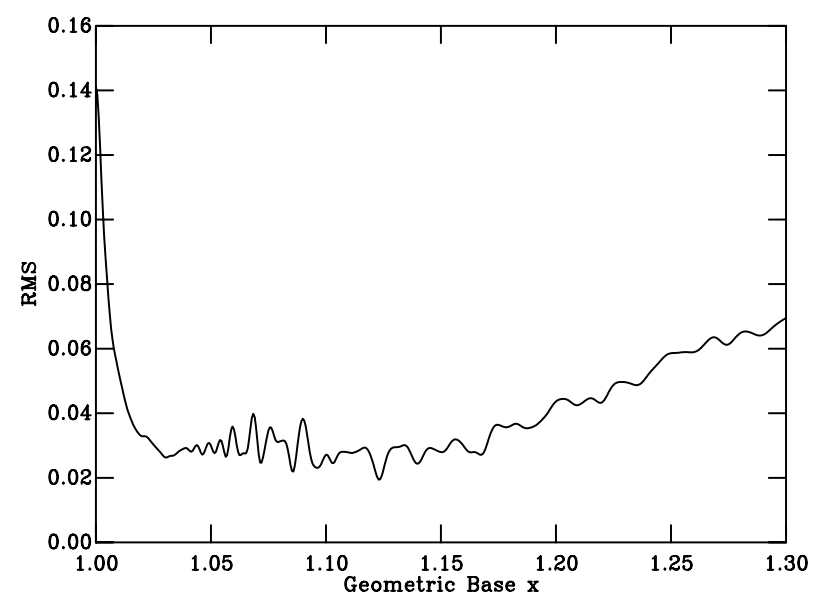

Fig. 4. Rms as a function of geometric base, for $N=25$. The optimum value is $x=1.124$.

knowledge of lower frequencies in order to do so. In particular, the precise frequency of the true signal is less clearly represented in the window function.

Note that the choice of geometric base is critical to the success of the sampling. Figure 5 shows how structure can be reintroduced into the window function by a poor choice of geometric base. This situation occurs because a sub-optimal geometric base essentially "wastes" information, oversampling some frequencies and undersampling others. In the pathological case of even sampling, every sampled frequency is a multiple of the true frequency, leading to massive redundancy. For datasets with more than 25 datapoints, the correct choice of geometric base is much more important. Figure 6 plots rms as a function of geometric base for datasets with 25, 100 and $500 \mathrm{ob}-$ servations. The larger datasets possess much narrower minima "valleys" than the $n=25$ dataset. At these dataset sizes, any substantial deviation from the optimal base will lead to rapid degradation of the window function.

\subsection{Noise}

Whilst in the idealised case without noise a suitable geometric sampling is the correct sampling, we must consider the effects of noise on the result. If noise dominates the periodogram, then 


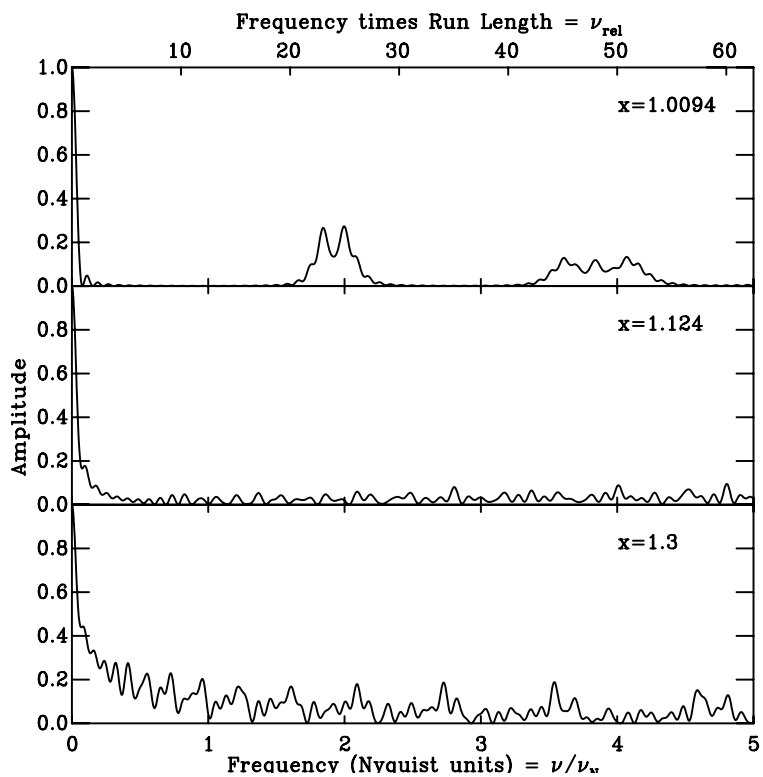

Fig. 5. Window functions for three geometric spacings of 25 datapoints. The top panel illustrates aliasing effects at a near linear sampling of $x=1.0094$, while at $x=1.3$ (bottom panel) the effects of near frequency interference are apparent. The optimum geometric spacing for this number of datapoints is shown for comparison $(x=1.124$, centre panel).

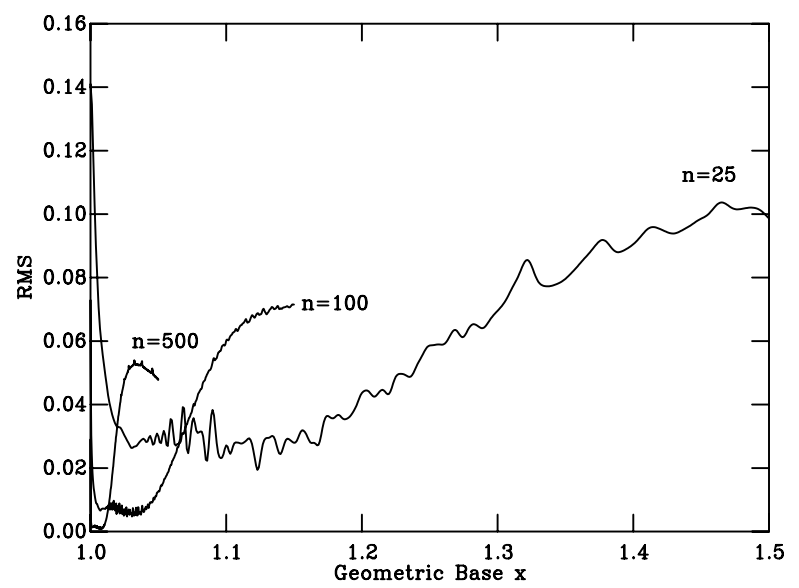

Fig. 6. Comparison of the variation of rms with geometric base, for three dataset sizes. When $n=25$, a relatively broad range of bases lie close to the minimum rms. For larger numbers of datapoints, the choice of optimal base is much more tightly constrained.

there may be little point in optimising in the manner described. A range of equally plausible "optimal" geometric bases will exist, since the quality of the final observed Fourier transform is noiselimited rather than window-function limited.

When making actual observations, an observer must decide how to divide a fixed total exposure time. This choice impacts signal-to-noise, since for most practical observing scenarios the signal-to-noise increases as the square root of the total exposure time. However, the window function has no noise dependency. The quality of a specific window function as a function of frequency is determined entirely by the choice of sampling time. This means that noise will only be of practical concern to the observing strategy if it is greater than the mean amplitude of what Deeming (1975) refers to as "near-frequency interference", and which we shall term spectral leakage.

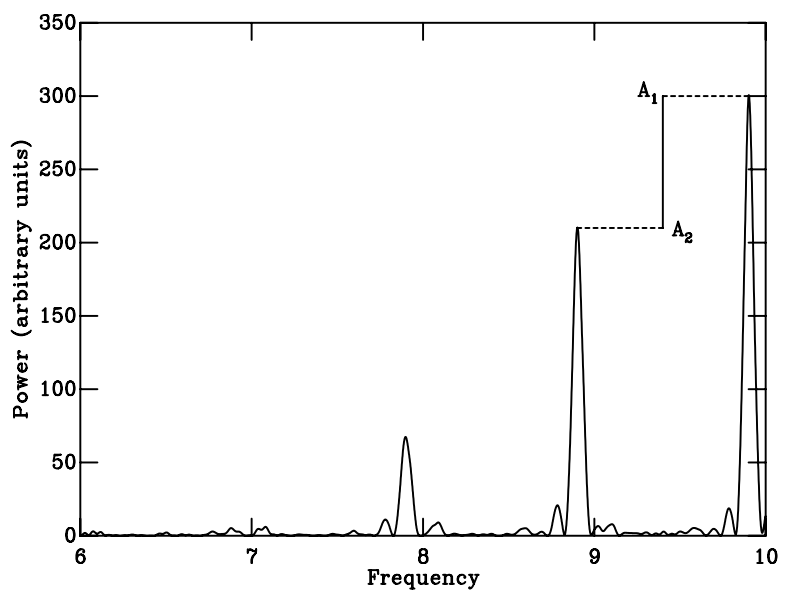

Fig. 7. Periodogram illustrating how the $A$ metric can be applied as a measure of relative alias strength. $A_{1}$ and $A_{2}$ are the amplitudes of the true peak (in this case, the highest peak) and the highest other peak in the transform. The ratio $\frac{A_{1}}{A_{2}}$ is our $A$ metric.

To demonstrate the practical effects of noise on our ability to recover a signal from a set of observations, we generate a set of artificial lightcurves, each a sinusoid of a single frequency, that span the range from 1-100 cycles per observing run. We define the relative period as the ratio of the period $P$ to the observing run $T$, such that

$P_{\text {rel }}=\frac{P}{T} \equiv \frac{1}{v_{\text {rel }}}$

Thus, we consider the range $0.01 \leq P_{\text {rel }} \leq 1$. For each generated period, we calculate the Scargle periodogram (Lomb 1976; Scargle 1982), as formulated by Horne \& Baliunas (1986).

The quality of the periodogram is assessed by calculating the peak ratio, $A$, which we define to be the ratio of the powers of the peak corresponding to the true period and the highest other peak in the periodogram. There are three regimes of interest for this metric. Figure 7 illustrates the "alias" regime, where the metric indicates the extent to which power has been lost to other strong signal candidates. When aliases have been largely suppressed or when the overall level of noise in the periodogram is large, the metric instead describes the prominence of the peak relative to the spectral leakage, or to the background noise. These two situations are illustrated in Figs. 8 and 9 respectively. The relationship to the alias property or peak amplitude leads us to denote this metric $A$.

The accuracy to which a period detection is required changes $A$. An observer would not be interested in all nearby peaks or sub-peaks, but the scale on which structure in the periodogram is important is obviously highly dependent on the nature of the science programme. For all our simulations, we consider periods within 10 per cent of the true period to be adequate, in line with typical accuracy requirements for rotation rate histograms (Littlefair et al. 2005). We assume that our periods have an infinite coherence length, i.e. that the phase information does not change over time.

For a 100 observation dataset, we apply noise equal to one third of the full amplitude of the signal (Fig. 10) and noise equal to the amplitude of the signal itself (Fig. 12). This yields a signalto-noise of 3.33 and 1 respectively. It should be noted that this is not the signal-to-noise normally quoted by astronomers, where the time-varying signal is superimposed upon a constant flux. For comparison, in a typical observing scenario where the full 


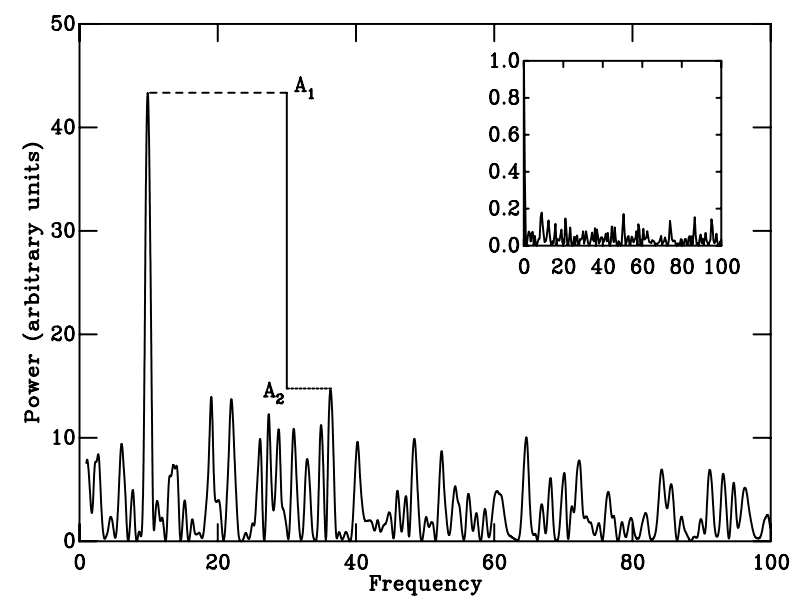

Fig. 8. Periodogram illustrating how the $A$ metric, defined as the ratio $\frac{A_{1}}{A_{2}}$, can represent the power at the true period relative to the spectral leakage. No noise has been added to this signal; interference effects are due to the (relatively poor) choice of sampling. The signal is a simple sinusoid with $P_{\text {rel }}=0.10163$, sampled with 100 datapoints using a geometric base of $x=1.10$, randomly respaced. The inset gives the amplitude of the corresponding window function, as a function of frequency.

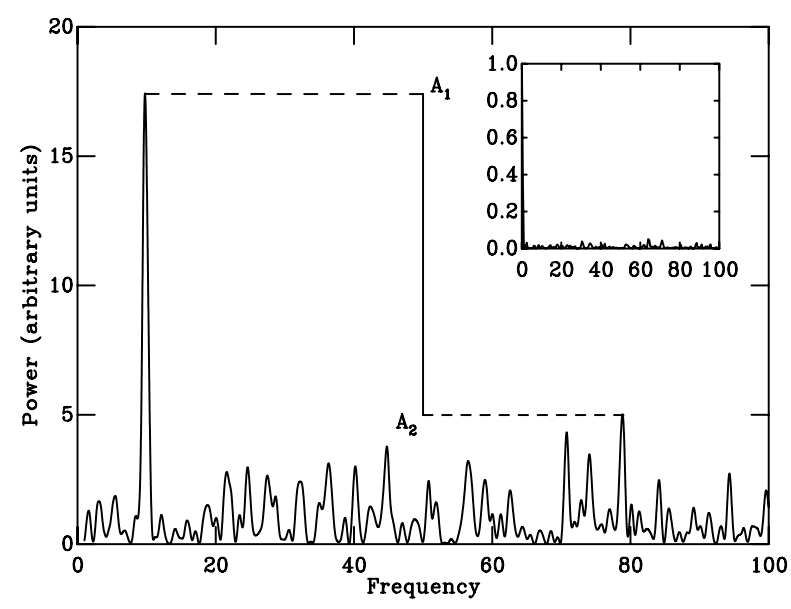

Fig. 9. Periodogram illustrating how the $A$ metric, defined as the ratio $\frac{A_{1}}{A_{2}}$, can represent the power at the true period relative to the overall level of noise present in the signal. The input signal is the same as for Fig. 8, but with the additional application of noise equal to half the amplitude of the signal. In this case, the geometric sampling base used was $x=1.03$, to minimise the effects of spectral leakage. The inset gives the amplitude of the corresponding window function, as a function of frequency; note the relatively minor interference contributions.

amplitude of the signal modulation might make up around 10 per cent of the incident signal flux, our simulated noise would correspond to an observational combined signal-to-noise of around 33 and 10.

In Fig. 10 the noise level is comparable to the spectral leakage, and we see the optimal base choice clearly picked out by the $A$ metric. For comparison, Fig. 11 gives the rms of the window function as a function of geometric base for the same dataset. Figure 12 illustrates the degradation of the periodogram under the effects of extreme noise. Under these conditions, the choice of geometric base is much less important, because the amplitude of the noise is typically much greater than the amplitude of the spectral leakage.

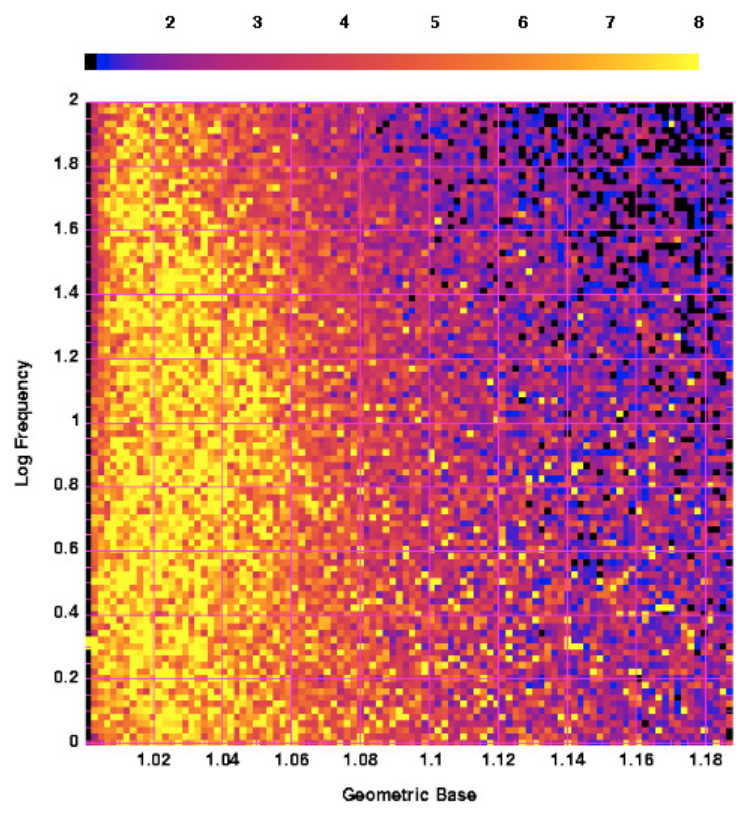

Fig. 10. $A$ for an $N=100$ ordered geometric lightcurve with noise equal to $30 \%$ of the signal amplitude, as a function of geometric base and injected signal frequency.

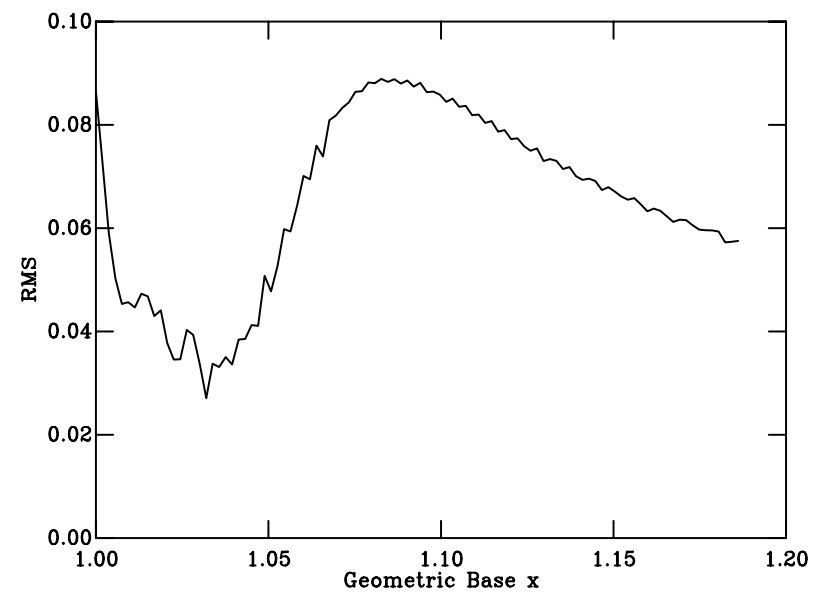

Fig. 11. Rms as a function of geometric base, for $N=100$. The optimum value is $x=1.032$.

We conclude that noise is not a contributing factor to the choice of optimal base for standard astronomical applications.

\subsection{Randomised geometric spacing}

Given the favourable properties of a standard geometric sampling, we can ask whether the order of the sampling matters. We can preserve our choice of spacing while modifying the order in which observations take place. This is equivalent to shuffling the gaps between observations. One motivation for doing this is that it allows individual observations to be placed with much more flexibility than an ordered geometric spacing, greatly easing scheduling problems.

Figure 13 plots the variation of rms with geometric base for a 25 point reshuffled geometric spacing. For comparison, the ordered geometric spacing is overplotted (dashes). In general, a randomised geometric spacing has a slightly higher rms than the equivalent ordered spacing over the range of optimal bases. Figure 14 shows the window function for the base with the 


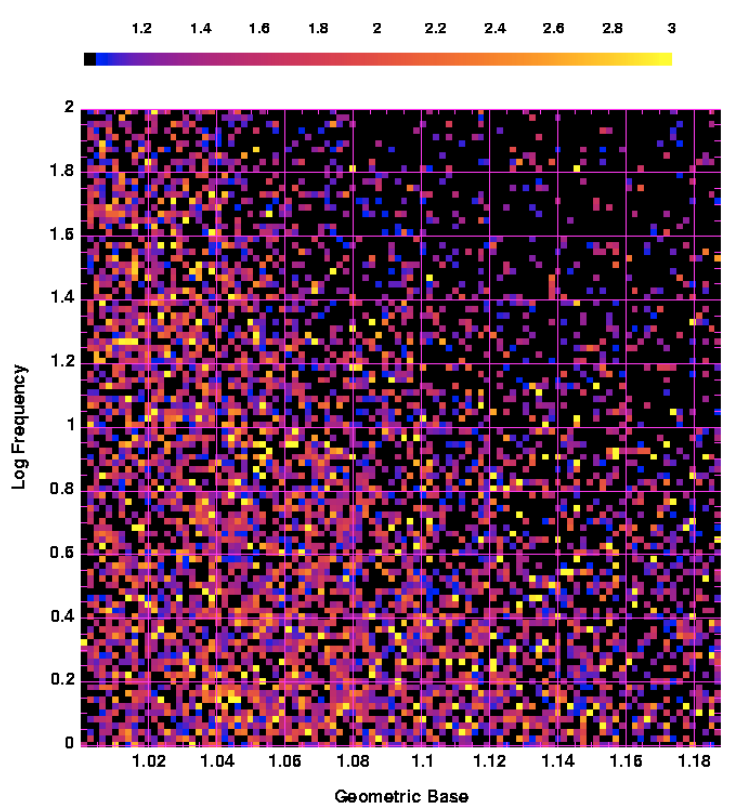

Fig. 12. $A$ for an $N=100$ ordered geometric lightcurve with noise equal to $100 \%$ of the signal amplitude, as a function of geometric base and injected signal frequency. High noise dominates the periodogram, washing out structure in the plane.

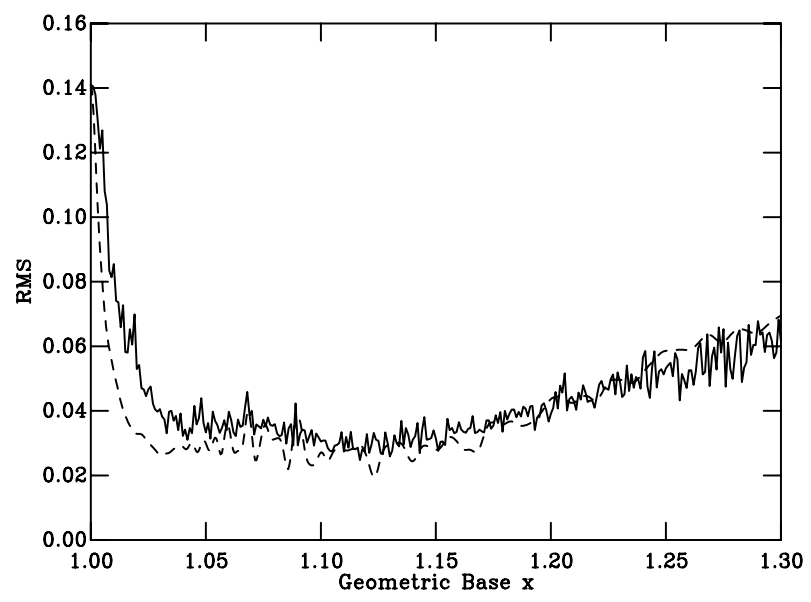

Fig. 13. rms as a function of randomised geometric base, for $N=25$ (solid line). For comparison, the ordered geometric spacing has been overplotted (dashes).

lowest rms. It should be noted that each random realisation has its own unique window function, and that for small values of $N$ the rms values can vary markedly from run to run. However, the range of variation in rms across the space of possible random realisations shrinks rapidly with increasing $N$.

The different behaviour between the ordered and randomised geometric spacing is more clearly illustrated in Fig. 15. The optimal range of bases remains almost the same as for the ordered case. In general, the smoothness (as measured by the rms value) of the window function of the best randomised geometric spacing is not quite as good as the equivalent ordered spacing. However, the randomised spacing degrades much more gracefully with increasing geometric base - for sub-optimal choices of the geometric base, a randomised spacing outperforms the ordered spacing. This has important implications for observing programmes in which the total number of observations is a highly dynamic quantity which cannot be accurately predicted in advance. In such a context, choosing a randomised geometric

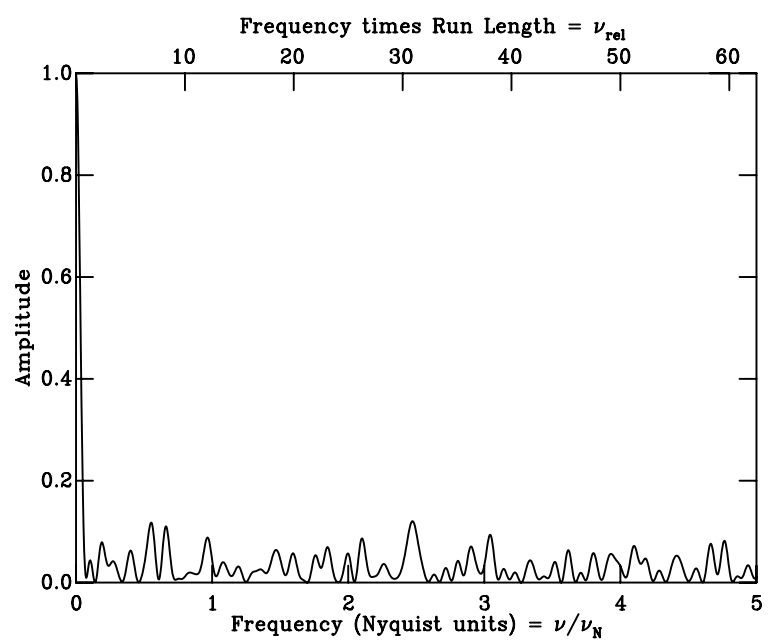

Fig. 14. A typical window function for an optimum randomised geometric sampling of 25 data points, where $x=1.128$. The rms is 0.025 .

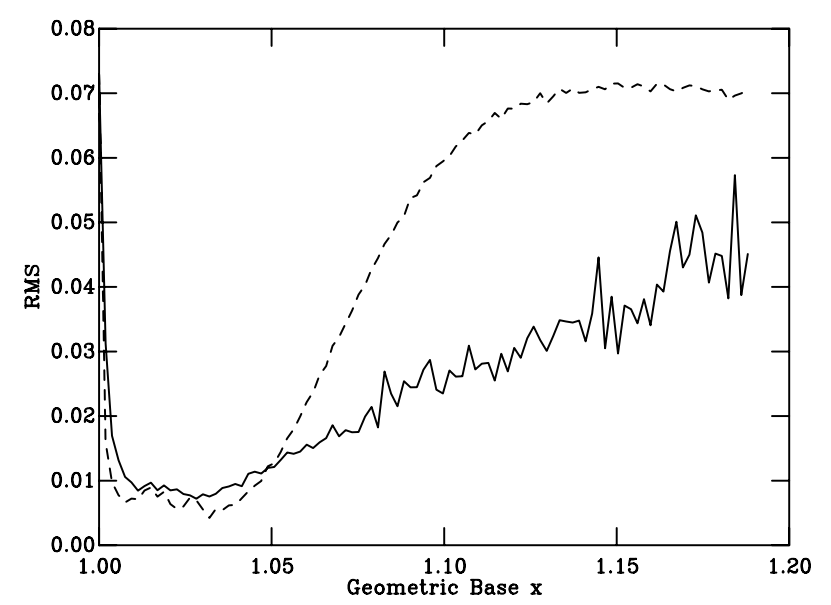

Fig. 15. Randomised geometric base as a function of rms, for $N=100$ (solid line). For comparison, the ordered geometric spacing has been overplotted (dashes).

spacing would allow the observing programme more flexibility with the total number of observations and their spacings, while seeking to optimise the schedule based on the current estimate of the run size and duration. Such an algorithm could form the basis of an adaptive dataset planning agent, an autonomous software entity which encapsulates sampling knowledge and attempts to exploit it in a real world environment.

\section{Practical application}

Figure 16 presents the optimal geometric base for a range of randomly respaced geometric sampling scenarios. By taking the period range and run-length of interest, converting them into a relative limiting frequency, and then estimating the total number of observations to be made, the ideal geometric base for different observation regimes can be found. Expressing the limiting frequency in units of $v_{\mathrm{N}}$, and substituting Eq. (1) gives

$\left(\frac{v}{v_{\mathrm{N}}}\right)=\frac{2 T v}{N}=\frac{2 v_{\mathrm{rel}}}{N}$

As an example, an astronomer with access to a robotic network is planning a 3 week observing run, searching for periods 


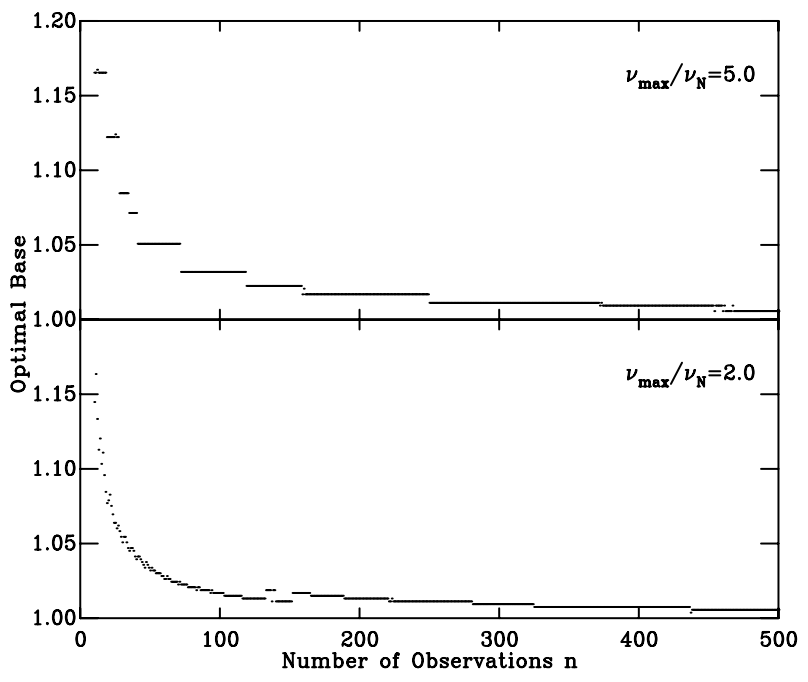

Fig. 16. Optimal geometric bases, as determined by minimum rms, for datasets spanning 10 to 500 datapoints. For a relative maximum limiting frequency of 2 (lower panel), optimal bases tend to be closer to linear sampling than an equivalent sampling extending over a much higher dynamic range (upper panel). Simulations out to a relative maximum limiting frequency of 12.5 show almost no deviation from the optimal bases calculated for the $v_{\max } / v_{\mathrm{N}}=5$ case.

ranging from $5 \mathrm{~h}$ to 10 days. This corresponds to a minimum relative period of 0.0099 , and thus a maximum limiting relative frequency $v_{\text {rel }}$ of 101 . If the total number of observations is likely to be around 100 , then $\left(v / v_{\mathrm{N}}\right) \approx 2$. Applying the lower curve in Fig. 16, the ideal sampling base is found to lie at around 1.02.

Although Fig. 16 has, in principle, to be recalculated for each particular value of $\left(v / \nu_{\mathrm{N}}\right)$, in practice we find no change in the set of optimum bases for relative maximum limiting frequencies greater than 5, and the optimal minima are sufficiently broad that crude interpolation should suffice for smaller values. If the relative limiting frequency is found to be below 1 , then enough datapoints exist to sample evenly - no further optimisation is required.

Thus the observer should apply the following procedure to build an observing schedule. The signal range of interest must be identified, and the likely size of the dataset and its duration estimated. This allows the optimal geometric base to be calculated. Having ascertained the optimal base, Eq. (5) may be used to calculate a series of observation times. The resulting gaps may then be reordered as desired as the run proceeds, for example to accommodate practical constraints such as weather conditions or telescope maintenance, or to facilitate a single night's intensive coverage during a period of dark time.

\section{Conclusions}

We have found the best solution to date for placing limited numbers of observations in such a way as to minimise aliasing effects arising from undersampling. By applying a simple geometric sampling rule, we are able to significantly outperform previous sampling schemes. Our scheme has the advantage that it is easy to apply at the telescope for datasets of arbitrary size, has calculated empirical solutions for different observing scenarios, and exhibits significant flexibility arising from the invariance of the optimal sampling choice under the effects of reordering. As Fig. 5 shows, by careful sampling the improvements that can be made in data quality, or alternatively, the savings that may be accrued in telescope time are substantial.

Acknowledgements. The authors would like to thank Charles Williams for many useful discussions. ESS is funded through a PPARC e-Science studentship. This work is part of the eSTAR project, which supports A.A. and which is jointly funded by the DTI, PPARC and EPSRC. We are grateful to the Starlink project for their flexibility in seconding A.A. to eSTAR. We are also grateful to the European Union VOTech project.

\section{References}

Allan, A., Naylor, T., Steele, I., et al. 2004, in Proc. SPIE, 5496, 313

Bode, M. F., Carter, D., \& Steele, I. A. 1999, Bulletin of the American Astronomical Society, 31, 1542

Chaplin, W. J., Elsworth, Y., Howe, R., et al. 1996, Sol. Phys., 168, 1

Deeming, T. J. 1975, Ap\&SS, 36, 137

Hessman, F. V., Gelderman, R., Naylor, T., Pennypacker, C., \& Steele, I. 2004, American Astronomical Society Meeting Abstracts, 205,

Horne, J. H., \& Baliunas, S. L. 1986, ApJ, 302, 757

Littlefair, S., Naylor, T., Burningham, B., \& Jeffries, R. D. 2005, MNRAS, 358, 341

Lomb, N. R. 1976, Ap\&SS, 39, 447

Mould, J. R., Hughes, S. M. G., Stetson, P. B., et al. 2000, ApJ, 528, 655

Nather, R. E., Winget, D. E., Clemens, J. C., Hansen, C. J., \& Hine, B. P. 1990 , ApJ, 361, 309

Scargle, J. D. 1982, ApJ, 263, 835

Schwarzenberg-Czerny, A. 2003, in Interplay of Periodic, Cyclic and Stochastic Variability in Selected Areas of the H-R Diagram, ASP Conf. Ser., 292, 383 